\title{
Modified two-step Simpson method for solving the fuzzy differential equations and the dependency problem
}

\author{
Kanagarajan K, Muthukumar S, Indrakumar S \\ Department of Mathematics, Sri Ramakrishna mission Vidyalaya College of Arts \& Science \\ Coimbatore-641020.
}

\begin{abstract}
In this paper, we study the numerical solution of fuzzy differential equations by using modified twostep Simpson method. This method is adopted to solve the dependency problem in fuzzy computation. Examples are presented to illustrate the computational aspects of this method.
\end{abstract}

Keywords: Fuzzy initial value problem, Dependency problem in fuzzy computation, Modified two-step method.

\section{Introduction}

Fuzzy Differential Equations (FDE) are used in modeling problems in science and engineering. Most of the problems in science and engineering require the solutions of FDE which are satisfied by fuzzy initial conditions, therefore a Fuzzy Initial Value Problem(FIVP) occurs and should be solved. Fuzzy set was first introduced by Zadeh[20]. Since then, the theory has been developed and it is now emerged as an independent branch of Applied Mathematics. The elementary fuzzy calculus based on the extension principle was studied by Dubois and Prade[11]. Seikkala[19] and Kaleva[12] have discussed FIVP. Buckley and Feuring[10] compared the solutions of FIVP which where obtained using different derivatives. The numerical solution of FIVP by Euler's method was studied by Ma et al.[14]. Abbasbandy and Allviranloo[1,2] proposed the Taylor method and the fourth order Runge-Kutta method for solving FIVP. Palligkinis et al.[17] applied the Runge-Kutta method for more general problems and proved the convergence for $n$-stage Runge-Kutta method. Mahmoud Mohseni Moghadam[15] studied the numerical solution of fuzzy differential equation by two-step method. The dependency problem in fuzzy computation was discussed by Ahmad and Hasan[4] and they used Euler's method based on Zadeh's extension principle for finding the numerical solution of FIVP. Recently they adopted the same computation method to derive the fourth order Runge-Kutta method[7] for FIVP. In this paper, we study the dependency problem in fuzzy computations by using Modified two-step method.

\section{Preliminary concepts}

In this section, we give some basic definitions and notations.

Definition 2.1. Subset $\widetilde{A}$ of a universal set $\mathrm{X}$ is said to be a fuzzy set if a membership function $\mu_{\widetilde{A}}(x)$ takes each object in $\mathrm{X}$ onto the interval $[0,1]$. The function $\mu_{\widetilde{A}}(x)$ is the possibility degrees to which each object is compatible with the properties that characterized the group.

A fuzzy set $\widetilde{A} \subseteq X$ can also be presented as a set of ordered pairs

$\widetilde{A}=\left\{\left(x, \mu_{\widetilde{A}}(x)\right): x \in X\right\}$.

The support, the core and the height of $\mathrm{A}$ are respectively

$$
\begin{aligned}
\operatorname{support}(\widetilde{A}) & =\left\{x \in X: x>\mu_{\widetilde{A}}(x)\right\}, \\
\operatorname{core}(\widetilde{A}) & =\left\{x \in X: \mu_{\widetilde{A}}(x)=1\right\}, \\
\operatorname{hgt}(\widetilde{A}) & =\sup _{x \in X} \mu_{\widetilde{A}}(x) .
\end{aligned}
$$

Definition 2.2. A fuzzy number is a convex fuzzy subset A of R, for which the following conditions are satisfied:

$$
\widetilde{A} \text { is normalized i.e. } \operatorname{hgt}(\widetilde{A})=1 \text {; }
$$

(ii) $\quad \mu_{\widetilde{A}}(x)$ are upper semicontinuous;

(iii) $\quad x \in R: \mu_{\widetilde{A}}(x)=\alpha$ are compact sets for $0<\alpha \leq 1$, and

$$
\overline{\left\{x \in R: \mu_{\widetilde{A}}(x) \geq \alpha\right\}} \text { are also compact sets for } 0<\alpha \leq 1 \text {. }
$$


Definition 2.3. If $\mathrm{F}(\mathrm{R})$ is the set of all fuzzy numbers, and $\widetilde{A} \in F(R)$, we can characterized $\widetilde{A}$ by its $\alpha$-levels by the following closed-bounded intervals:

$$
\begin{aligned}
& {[\widetilde{A}]^{\alpha}=\left\{x \in R: \mu_{\widetilde{A}}(x) \geq \alpha\right\}=\left[a_{1}^{\alpha}, a_{2}^{\alpha}\right], \quad 0<\alpha \leq 1,} \\
& {[\widetilde{A}]^{\alpha}=\left\{\overline{x \in R: \mu_{\widetilde{A}}(x) \geq \alpha}\right\}=\left[\overline{a_{1}^{\alpha}, a_{2}^{\alpha}}\right], \quad 0<\alpha \leq 1 \text {. }}
\end{aligned}
$$

Operation on fuzzy numbers can be described as follows: If $\widetilde{A}, \widetilde{B} \in F(R)$, then for $0<\alpha \leq 1$

1. $[\widetilde{A}+\widetilde{B}]^{\alpha}=\left[a_{1}^{\alpha}+b_{1}^{\alpha}, a_{2}^{\alpha}+b_{2}^{\alpha}\right] ;[\widetilde{A}-\widetilde{B}]^{\alpha}=\left[a_{1}^{\alpha}-b_{1}^{\alpha}, a_{2}^{\alpha}-b_{2}^{\alpha}\right] ;$

2. $[\widetilde{A} \cdot \widetilde{B}]^{\alpha}=\left[\min \left\{a_{1}^{\alpha} \cdot b_{1}^{\alpha}, a_{1}^{\alpha} \cdot b_{2}^{\alpha}, a_{2}^{\alpha} \cdot b_{1}^{\alpha}, a_{2}^{\alpha} \cdot b_{2}^{\alpha}\right\}, \max \left\{a_{1}^{\alpha} \cdot b_{1}^{\alpha}, a_{1}^{\alpha} \cdot b_{2}^{\alpha}, a_{2}^{\alpha} \cdot b_{1}^{\alpha}, a_{2}^{\alpha} \cdot b_{2}^{\alpha}\right\}\right]$;

3. $\left[\frac{\widetilde{A}}{\widetilde{B}}\right]^{\alpha}=\left[\min \left\{\frac{a_{1}^{\alpha}}{b_{1}^{\alpha}}, \frac{a_{1}^{\alpha}}{b_{2}^{\alpha}}, \frac{a_{2}^{\alpha}}{b_{1}^{\alpha}}, \frac{a_{2}^{\alpha}}{b_{2}^{\alpha}}\right\}, \max \left\{\frac{a_{1}^{\alpha}}{b_{1}^{\alpha}}, \frac{a_{1}^{\alpha}}{b_{2}^{\alpha}}, \frac{a_{2}^{\alpha}}{b_{1}^{\alpha}}, \frac{a_{2}^{\alpha}}{b_{2}^{\alpha}}\right\}\right]$ here $0 \notin[\widetilde{B}]^{\alpha}$;

4. $\quad[s \widetilde{A}]^{\alpha}=s[\widetilde{A}]^{\alpha}$; where $s$ is sclar and

5. $\quad\left[a_{1}^{\alpha_{i}}, b_{1}^{\alpha_{i}}\right] \subset\left\lfloor a_{1}^{\alpha_{j}}, b_{1}^{\alpha_{j}}\right\rfloor$ for $0<\alpha_{i} \leq \alpha_{j}$.

Definition 2.4. A fuzzy process is a mapping $\tilde{x}: I \rightarrow F(R)$, where I is a real interval [19]. This process can be denoted as:

$[\widetilde{x}(t)]^{\alpha}=\left[\tilde{x}_{1}^{\alpha}(t), \tilde{x}_{2}^{\alpha}(t)\right], \quad t \in I \quad$ and $\quad 0<\alpha \leq 1$.

The fuzzy derivative of a fuzzy process $\mathrm{x}(\mathrm{t})$ is defined by

$\left[\widetilde{x}^{\prime}(t)\right]^{\alpha}=\left[\widetilde{x}_{1}^{\prime \alpha}(t), \widetilde{x}_{2}^{\prime \alpha}(t)\right], \quad t \in I \quad$ and $\quad 0<\alpha \leq 1$.

\section{Fuzzy Initial value problem}

Consider the fuzzy initial value problem

$$
\frac{d x(t)}{d t}=f(t, x(t)), \quad x(0)=\tilde{X}_{0},
$$

where $f: R_{+} \times R \rightarrow R$ is a continuous mapping and $\widetilde{X}_{0} \in F(R)$ with $\alpha$-level interval

$\left[\widetilde{x}_{0}\right]^{\alpha}=\left[\widetilde{x}_{01}^{\alpha}, \widetilde{x}_{02}^{\alpha}\right], \quad 0<\alpha \leq 1$.

When $x=x(t)$ is a fuzzy number, the extension principle of Zadeh leads to the following definition:

$f(t, x)(s)=\sup \{x(\tau): f(t, \tau)\}, \quad s \in R$.

It follows that

$[f(t, x(t))]^{\alpha}=\left[f_{1}^{\alpha}(t, x(t)), f_{2}^{\alpha}(t, x(t))\right], \quad 0<\alpha \leq 1$,

where

$f_{1}^{\alpha}(t, x(t))=\min \left\{f(t, w): w \in\left[x_{1}^{\alpha}(t), x_{2}^{\alpha}(t)\right]\right\}, \quad 0<\alpha \leq 1$,

$f_{2}^{\alpha}(t, x(t))=\max \left\{f(t, w): w \in\left[x_{1}^{\alpha}(t), x_{2}^{\alpha}(t)\right]\right\}, \quad 0<\alpha \leq 1$.

Theorem 3.1. Let $\mathrm{f}$ satisfy

$$
\left|f(t, x)-f\left(t, x^{*}\right)\right| \leq g\left(t,\left|x-x^{*}\right|\right), \quad t \geq 0, \quad x, x^{*} \in R,
$$

where $g: R_{+} \times R_{+} \rightarrow R_{+}$is a continuous mapping such that $r \rightarrow g(t, r)$ is nondecreasing, 0the IVP

$$
z(t)=g(t, z(t)), \quad z(0)=z_{0},
$$

has a solution on $R_{+}$for $z_{0}>0$ and that $z(t) \equiv 0$ is the only solution of equation (15) for $z_{0}=0$. Then the FIVP (10) has a unique fuzzy solution.

Proof: See [19].

In the fuzzy computation, the dependency problem arises when we apply the straightforward fuzzy interval arithmetic and Zadeh's extension principle by computing the interval separately. This will affect errors in the approximations of some numerical computations; in the end, these possible errors can make the results be far from the correct results [8].

We now explain the dependency problem concept, consider the real value function

$$
f(x)=2 x^{2}+5 x+2 \text {. }
$$


For a fuzzy number $[\tilde{X}]^{\alpha}=[2 \alpha-2,2-2 \alpha]$ two common ways can be applied to obtain $f(\tilde{X})$. First, the straightforward fuzzy interval arithmetic:

$$
\begin{aligned}
f\left([\widetilde{X}]^{\alpha}\right)= & 2[2 \alpha-2,2-2 \alpha]^{2}+5[2 \alpha-2,2-2 \alpha]+2 \\
= & 2\left[\min \left\{(2 \alpha-2)^{2},(2 \alpha-2)(2-2 \alpha),(2-2 \alpha)^{2}\right\},\right. \\
& \left.\quad \max \left\{(2 \alpha-2)^{2},(2 \alpha-2)(2-2 \alpha),(2-2 \alpha)^{2}\right\}\right] \\
+ & 5[2 \alpha-2,2-2 \alpha]+2 .
\end{aligned}
$$

If $\alpha=0$, we get

$$
f\left([\tilde{X}]^{0}\right)=[-16,20] \text {. }
$$

The second is by applying Zadeh's extension principle separately, that is:

$$
f\left([\tilde{X}]^{\alpha}\right)=2 X^{2}+5 X+2 \text {. }
$$

For $\alpha=0$, the solution is hence:

$$
f\left([\tilde{X}]^{0}\right)=[-8,20] \text {. }
$$

The solutions of equation (19) and (21) are not the correct range of $[\tilde{X}]^{0}$. The correct one can be obtained by assuming the right hand side of equation (20) as one expression and then applying Zadeh's extension principle. According to this, the correct range is:

$$
f\left([\widetilde{X}]^{0}\right)=\left[-\frac{9}{8}, 20\right] \text {. }
$$

Ahmad and Hasan in [5] developed a new computation method that can reduce the computational complexity and overestimation in the results. This method is based on the incorporation of the optimization technique into Zadeh's extension principle. This techniques requires the creation of partition on a fuzzy interval, which is defined as follows.

Let $\widetilde{X}$ be a triangular fuzzy number defined by the three numbers $a_{1}<a_{2}<a_{3}$, with its graph having a triangular base on the interval $\left[a_{1}, a_{3}\right]$. For $0<\alpha \leq 1$, let $[\widetilde{x}(t)]^{\alpha}=\left[x_{1}^{\alpha}(t), x_{2}^{\alpha}(t)\right]$ be the $\alpha$-level interval. To make the partition for this fuzzy interval, we divide this interval into subintervals using the discrete set of points $0=\alpha_{0}<\alpha_{1}<\cdots<\alpha_{n}=1$ in $[0,1]$, where $\alpha_{i}-\alpha_{i-1}=k=\frac{1}{n}, i=1, \ldots, n$.

For every $\alpha_{i}, i=0, \ldots, n$, we get

$$
[\tilde{X}]^{\alpha_{i}}=\left[x_{1}^{\alpha_{i}}, x_{1}^{\alpha_{i+1}}\right] \bigcup\left[x_{1}^{\alpha_{i+1}}, x_{1}^{\alpha_{n 1}}\right] \bigcup\left[x_{1}^{\alpha_{n}}, x_{2}^{\alpha_{n}}\right] \bigcup\left[x_{2}^{\alpha_{n i}}, x_{2}^{\alpha_{i+1}}\right] \bigcup\left[x_{2}^{\alpha_{i+1}}, x_{2}^{\alpha_{1}}\right],
$$

where in the case of triangular fuzzy number, $x_{1}^{\alpha_{n}}=x_{2}^{\alpha_{n}}$.

To compute $\tilde{Y}=f(\tilde{X})$ where $\mathrm{f}$ is a real continuous function and $\tilde{X}$ is a fuzzy number (as a triangular), we need to do it at each possibility level as follows:

$$
\begin{aligned}
& y_{1}^{\alpha_{i}}=\min \left[\min _{x \in\left[x_{1}^{\alpha_{i}}, x_{1}^{\alpha_{i+1}}\right]} f(x), \ldots, \min _{x \in\left[x_{1}^{\alpha_{n}}, x_{2}^{\alpha_{n}}\right]} f(x), \ldots \min _{x \in\left[x_{2}^{\alpha_{i+1}}, x_{2}^{\alpha_{1}}\right]} f(x)\right], \\
& y_{2}^{\alpha_{i}}=\max \left[\max _{x \in\left[x_{1}^{\alpha_{i}}, x_{1}^{\alpha_{i+1}}\right]} f(x), \ldots, \max _{x \in\left[x_{1}^{\alpha_{n}}, x_{2}^{\alpha_{n}}\right]} f(x), \ldots \max _{x \in\left[x_{2}^{\alpha_{i+1}}, x_{2}^{\alpha_{1}}\right]} f(x)\right],
\end{aligned}
$$

where $y_{1}^{\alpha_{i}}$ and $y_{2}^{\alpha_{i}}$ are bounded values of $[\widetilde{Y}]^{\alpha_{i}}$ for $i=1, \ldots, n$. The optimization problems in equation (24) and (25) were computed and analytically discussed in [6] by using Brent's method [6]. Ahmad and Hasan [9] also proposed a new strategy to compute these optimization problems. The new computation method has a low complexity and has the ability to reduce the overestimation in the obtaining results. Ahmad and Hasan [4] applied this approach to extend the classical Euler's method. They compared their method with that of $\mathrm{Ma}$ et al.[14], where the results showed that there is overestimation in the computation using the latter method and the approximate solution does not converge to the exact solution. It diverged when time increases. 


\section{A Modified two-step simpson method}

To obtain the Simpson method for numerical solution of the system (9), we integrate the system from $t_{r-1}$ to $t_{r+1}$ and use the Simpson method for right hand side of

$\int_{t_{r-1}}^{t_{r+1}} x^{\prime}(s) d s=\int_{t_{r-1}}^{t_{r+1}} f(s, x(s)) d s$.

Therefore we have,

$$
\begin{aligned}
x\left(t_{r+1}\right)-x\left(t_{r-1}\right) & =\frac{h}{3}\left[f\left(t_{r-1}, x\left(t_{r-1}\right)\right)+4 f\left(t_{r}, x\left(t_{r}\right)\right)+f\left(t_{r+1}, x\left(t_{r+1}\right)\right)\right], \\
x\left(t_{r+1}\right) & =x\left(t_{r-1}\right)+\frac{h}{3}\left[f\left(t_{r-1}, x\left(t_{r-1}\right)\right)+4 f\left(t_{r}, x\left(t_{r}\right)\right)+f\left(t_{r+1}, x\left(t_{r+1}\right)\right)\right] .
\end{aligned}
$$

Equation (27) is an implicit equation in term of $x\left(t_{r+1}\right)$. To avoid of solving such implicit equation, we will substitute $x\left(t_{r+1}\right)$ by $x\left(t_{r}\right)+h f\left(t_{r}, x\left(t_{r}\right)\right)+\frac{h^{2}}{2} f^{\prime}\left(t_{r}, x\left(t_{r}\right)\right)$ in right hand side of (27). But we have

$$
\begin{aligned}
f\left(t_{r+1}, x\left(t_{r}\right)\right. & \left.+h f\left(t_{r}, x\left(t_{r}\right)\right)+\frac{h^{2}}{2} f^{\prime}\left(t_{r}, x\left(t_{r}\right)\right)\right) \\
= & f\left(t_{r+1}, x\left(t_{r}\right)+h f\left(t_{r}, x\left(t_{r}\right)\right)+\frac{h^{2}}{2} f^{\prime}\left(t_{r}, x\left(t_{r}\right)\right) f_{x}\left(t_{r+1}, x\left(t_{r}\right)\right)\right),
\end{aligned}
$$

where $t_{0} \leq t_{1} \leq \cdots \leq t_{N}=T$ and $h=\frac{T-t_{0}}{N}=t_{r+1}-t_{r}, r=0,1, \ldots, N$.

For the fuzzy initial condition of equation (9), we modify the implicit modified Simpson method by taking into account the dependency problem in fuzzy computation. We consider the right-hand side of equation (27) as one function

$$
V\left(t_{r}, h, x_{r}\right)=x\left(t_{r-1}\right)+\frac{h}{3}\left[f\left(t_{r-1}, x\left(t_{r-1}\right)\right)+4 f\left(t_{r}, x\left(t_{r}\right)\right)+f\left(t_{r+1}, x\left(t_{r}\right)+h f\left(t_{r}, x\left(t_{r}\right)\right)\right)\right]
$$

or by the equivalent formula

$$
V\left(t_{r}, h, x_{r}\right)=x\left(t_{r-1}\right)+\frac{h}{3}\left\{\begin{array}{l}
f\left(t_{r-1}, x_{r-1}\right)+4 f\left(t_{r}, x_{r-1}+h f\left(t_{r-1}, x_{r-1}\right)+\frac{h^{2}}{2} f^{\prime}\left(t_{r-1}, x_{r-1}\right)\right) \\
+f\left(t_{r+1}, x_{r-1}\right)+h f\left(t_{r-1}, x\left(t_{r-1}\right)\right)+\frac{h^{2}}{2} f^{\prime}\left(t_{r-1}, x_{r-1}\right) \\
\left.+h f\left(t_{r}, t_{r+1}, x_{r-1}+h f\left(t_{r-1}, x\left(t_{r-1}\right)\right)+\frac{h^{2}}{2} f^{\prime}\left(t_{r-1}, x_{r-1}\right)\right)\right),
\end{array}\right.
$$

Now, let $\widetilde{X} \in F(R)$, the formula

$$
V\left(t_{r}, h, \tilde{X}_{r}\right)\left(v_{r}\right)=\left\{\begin{array}{ccc}
\sup _{x_{r} \in V^{-1}\left(t_{r}, h, v_{r}\right)} \tilde{X}_{r}\left(x_{r}\right), & \text { if } & v_{r} \in \operatorname{range}(V) ; \\
0, & \text { if } & v_{r} \notin \operatorname{range}(V)
\end{array}\right.
$$

can extend equation (30) in the fuzzy setting.

Let $\left[\tilde{X}_{r}\right]^{\alpha}=\left[x_{r, 1}^{\alpha}, x_{r, 2}^{\alpha}\right]$ represent the $\alpha$-level of the fuzzy number defined in equation (31). We rewrite equation (31) using the $\alpha$-level as follows:

$V\left(t_{r}, h,\left[\tilde{X}_{r}\right]^{\alpha}\right)=\left[\min \left\{V\left(t_{r}, h, x\right) \mid x \in\left[x_{r, 1}^{\alpha}, x_{r, 2}^{\alpha}\right]\right\}, \max \left\{V\left(t_{r}, h, x\right) \mid x \in\left[x_{r, 1}^{\alpha}, x_{r, 2}^{\alpha}\right]\right\}\right]$.

By applying equation (32) in (27) we get

$\left[\widetilde{X}_{r+1}\right]^{\alpha}=\left[x_{r+1,1}^{\alpha}, x_{r+1,2}^{\alpha}\right]$,

where

$x_{r+1,1}^{\alpha}=\min \left\{V(t, h, x) \mid x \in\left[x_{r, 1}^{\alpha}, x_{r, 2}^{\alpha}\right]\right\}$, 
$x_{r+1,2}^{\alpha}=\max \left\{V(t, h, x) \mid x \in\left[x_{r, 1}^{\alpha}, x_{r, 2}^{\alpha}\right]\right\}$.

or

$$
\begin{aligned}
& x_{r+1,1}^{\alpha}=\min \left\{x\left(t_{r-1}\right)+\frac{h}{3}\left[f\left(t_{r-1}, x\left(t_{r-1}\right)\right)+4 f\left(t_{r}, x\left(t_{r}\right)\right)+f\left(t_{r+1}, x\left(t_{r+1}\right)\right)\right] \mid x \in\left[x_{r, 1}^{\alpha}, x_{r, 2}^{\alpha}\right]\right\}, \\
& x_{r+1,2}^{\alpha}=\max \left\{x\left(t_{r-1}\right)+\frac{h}{3}\left[f\left(t_{r-1}, x\left(t_{r-1}\right)\right)+4 f\left(t_{r}, x\left(t_{r}\right)\right)+f\left(t_{r+1}, x\left(t_{r+1}\right)\right)\right] \mid x \in\left[x_{r, 1}^{\alpha}, x_{r, 2}^{\alpha}\right]\right\} .
\end{aligned}
$$

By using the computational method proposed in [5], we compute the minimum and maximum in equations (36), (37) as follows:

$$
\begin{aligned}
& x_{r+1,1}^{\alpha_{i}}=\min \left[\min _{x \in\left[x_{1}^{\alpha_{i}}, x_{1}^{\alpha_{i+1}}\right]} V(t, h, x), \ldots, \min _{x \in\left[x_{1}^{\alpha_{n}}, x_{2}^{\alpha_{n}}\right]} V(t, h, x), \ldots, \min _{x \in\left[x_{2}^{\alpha_{i+1}}, x_{2}^{\alpha_{1}}\right]} V(t, h, x)\right], \\
& x_{r+1,2}^{\alpha_{i}}=\max \left[\max _{x \in\left[x_{1}^{\alpha_{i}}, x_{1}^{\alpha_{i+1}}\right]} V(t, h, x), \ldots, \max _{x \in\left[x_{1}^{\alpha_{n}}, x_{2}^{\alpha_{n}}\right]} V(t, h, x), \ldots, \max _{x \in\left[x_{2}^{\alpha_{i+1}}, x_{2}^{\alpha_{1}}\right]} V(t, h, x)\right] .
\end{aligned}
$$

\section{Numerical Examples}

In this section, we consider two examples to solve FDE under Seikkala's differentiability [19].

Example 5.1. Consider the following FIVP [7].

$$
\left\{\begin{array}{cl}
x^{\prime}(t)=x(1-2 t), & t \in[0,2] ; \\
\tilde{X}^{0}(w)=\left\{\begin{array}{cl}
0, & \text { if } w<-0.5 ; \\
1-4 w^{2}, & \text { if }-0.5 \leq w \leq 0.5 ; \\
0, & \text { if } w>0.5 ;
\end{array}\right.
\end{array}\right.
$$

The exact solution of equation (40) is given by

$$
[X(t)]^{\alpha}=\left[\left(-\frac{\sqrt{(1-\alpha)}}{2}\right) e^{t-t^{2}},\left(\frac{\sqrt{(1-\alpha)}}{2}\right) e^{t-t^{2}}\right] .
$$

\begin{tabular}{|c|c|c|c|c|c|c|c|c|c|c|}
\hline \multirow{2}{*}{$\alpha$} & \multicolumn{2}{|c|}{$\mathrm{AB} 2$} & \multicolumn{2}{|c|}{ Modified 2-step } & \multicolumn{2}{|c|}{ Exact } & \multicolumn{2}{|c|}{ Error AB2 } & \multicolumn{2}{|c|}{ Error Modified 2-step } \\
\hline & $x_{1}(t ; \alpha)$ & $x_{2}(t ; \alpha)$ & $x_{1}(t ; \alpha)$ & $x_{2}(t ; \alpha)$ & $x_{1}(t ; \alpha)$ & $x_{2}(t ; \alpha)$ & $x_{1}(t ; \alpha)$ & $x_{2}(t ; \alpha)$ & $x_{1}(t ; \alpha)$ & $x_{2}(t ; \alpha)$ \\
\hline 0.0 & -0.0668 & 0.0668 & -0.0673 & 0.0673 & -0.0677 & 0.0677 & $-8.01 \mathrm{e}-4$ & $-8.01 \mathrm{e}-4$ & $-3.42 \mathrm{e}-4$ & $-3.42 \mathrm{e}-4$ \\
\hline 0.1 & -0.0634 & 0.0634 & -0.0638 & 0.0638 & -0.0642 & 0.0642 & $-7.60 \mathrm{e}-4$ & $-7.60 \mathrm{e}-4$ & $-3.25 e-4$ & $-3.25 e-4$ \\
\hline 0.2 & -0.0598 & 0.0598 & -0.0602 & 0.0602 & -0.0605 & 0.0605 & $-7.16 \mathrm{e}-4$ & $-7.16 \mathrm{e}-4$ & $-3.06 \mathrm{e}-4$ & $-3.06 \mathrm{e}-4$ \\
\hline 0.3 & -0.0559 & 0.0559 & -0.0563 & 0.0563 & -0.0566 & 0.0566 & $-6.70 e-4$ & $-6.70 e-4$ & $-2.86 \mathrm{e}-4$ & $-2.86 \mathrm{e}-4$ \\
\hline 0.4 & -0.0517 & 0.0517 & -0.0521 & 0.0521 & -0.0524 & 0.0524 & $-6.20 \mathrm{e}-4$ & $-6.20 \mathrm{e}-4$ & $-2.65 e-4$ & $-2.65 e-4$ \\
\hline 0.5 & -0.0472 & 0.0472 & -0.0476 & 0.0476 & -0.0478 & 0.0478 & $-5.66 \mathrm{e}-4$ & $-5.66 \mathrm{e}-4$ & $-2.42 \mathrm{e}-4$ & $-2.42 \mathrm{e}-4$ \\
\hline 0.6 & -0.0422 & 0.0422 & -0.0425 & 0.0425 & -0.0428 & 0.0428 & $-5.06 \mathrm{e}-4$ & $-5.06 e-4$ & $-2.16 \mathrm{e}-4$ & $-2.16 \mathrm{e}-4$ \\
\hline 0.7 & -0.0366 & 0.0366 & -0.0368 & 0.0368 & -0.0371 & 0.0371 & $-4.38 \mathrm{e}-4$ & $-4.38 \mathrm{e}-4$ & $-1.87 \mathrm{e}-4$ & $-1.87 \mathrm{e}-4$ \\
\hline 0.8 & -0.0299 & 0.0299 & -0.0301 & 0.0301 & -0.0303 & 0.0303 & $-3.58 \mathrm{e}-4$ & $-3.58 \mathrm{e}-4$ & $-1.53 e-4$ & $-1.53 e-4$ \\
\hline 0.9 & -0.0211 & 0.0211 & -0.0212 & 0.0212 & -0.0214 & 0.0214 & $-2.53 e-4$ & $-2.53 e-4$ & $-1.08 \mathrm{e}-4$ & $-1.08 \mathrm{e}-4$ \\
\hline 1.0 & 0.0000 & 0.0000 & 0.0000 & 0.0000 & 0.0000 & 0.0000 & 0.0000 & 0.0000 & 0.0000 & 0.0000 \\
\hline
\end{tabular}

The approximate fuzzy solution by proposed modified two-step Simpson method, the absolute results of the numerical fuzzy modified two-step Simpson method approximated solutions at $t_{20}=2$. See Table 1 and Figure 1 and 2.

Table 1.The error of the obtained results with the exact solution at $\mathrm{t}=2$. 


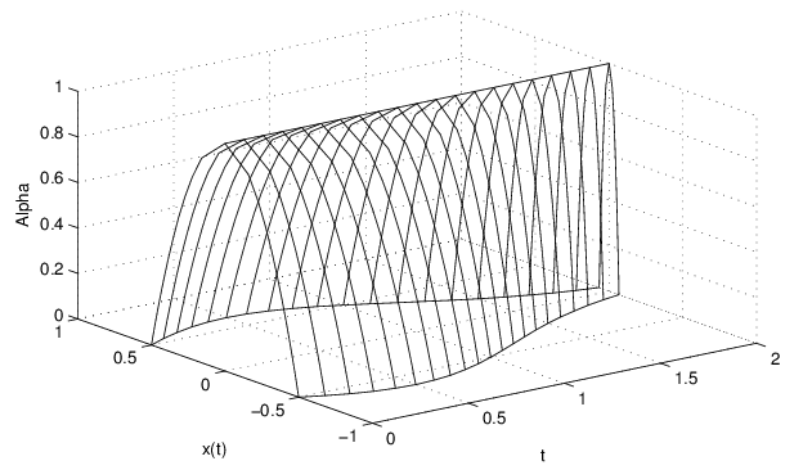

Fig 1: The approximation of fuzzy solution by modified two-step Simpson method $(\mathrm{h}=0.1)$

The approximation fuzzy solutions by the proposed two-step method and Adams-Bashforth method of order two (AB2) are given by Figure 1 and 2 respectively.
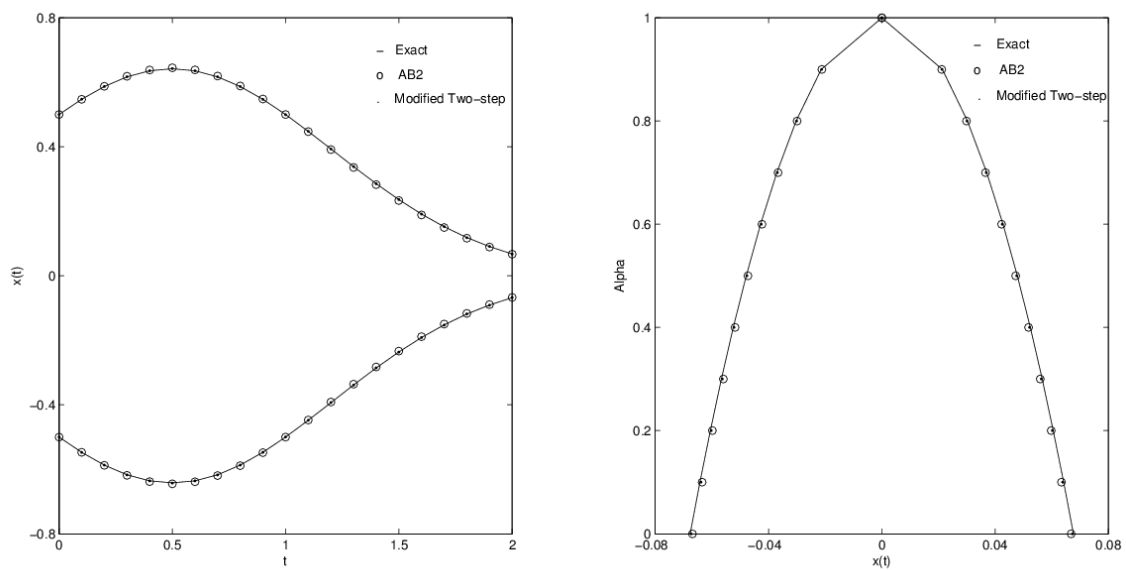

Fig 2 : Comparison between the exact, AB2, modified two-step Simpson method

For this example, the comparison of the absolute local error between two-step method, AdamsBashforth method of order two and with the fuzzy exact solution is given in Table 1 for various values of $\alpha$ level $(\alpha=0,0.1, \ldots 0.9,1)$ and fixed value of $t_{20}=2$. The results show that two-step method is accurate than Adams-Bashforth method of order two. The graphical comparison of a fuzzy solution between the two-step method, Adams-Bashforth method of order two, and the exact solutions at fixed $\left(t_{10}=1\right)$ in figure 2(b). The behavior solutions of the end points of the fuzzy intervals of a fuzzy exact solution and of Two-step method and Adams-Bashforth method of order two fuzzy approximated solutions are plotted and compared in Fig 2(a) at $\alpha_{1}=0$. Fig 2 clearly shows that two-step method provides more accurate results than Adams-Bashforth method of order two.

Example 5.2. Consider the following FIVP.

$$
\left\{\begin{array}{cc}
x^{\prime}(t)=x\left(t^{2}-4 t+3\right), & t \in[0,2] ; \\
0, & \text { if } w<-0.5 ; \\
\tilde{X}^{0}(w)=\left\{\begin{array}{cl}
\text { if }-0.5 \leq w \leq 0.5 ; \\
1-4 w^{2}, & \text { if } w>0.5 ;
\end{array}\right.
\end{array}\right.
$$

The exact solution of equation (42) is given by

$[X(t)]^{\alpha}=\left[\left(-\frac{\sqrt{(1-\alpha)}}{2}\right) e^{\frac{t^{3}}{3}-2 t^{2}+3},\left(\frac{\sqrt{(1-\alpha)}}{2}\right) e^{\frac{t^{3}}{3}-2 t^{2}+3}\right]$.

The approximate fuzzy solutions by proposed modified two-step Simpson method, the absolute results of the 
modified two-step Simpson method approximated solutions at $t_{20}=2$. See Table 2 and Figure 3 and 4 .

Table 2. The error of the obtained results with the exact solution at $\mathrm{t}=2$.

\begin{tabular}{|c|c|c|c|c|c|c|c|c|c|c|}
\hline \multirow{2}{*}{$\alpha$} & \multicolumn{2}{|c|}{$\mathrm{AB} 2$} & \multicolumn{2}{|c|}{ Modified 2-step } & \multicolumn{2}{|c|}{ Exact } & \multicolumn{2}{|c|}{ Error $\mathrm{AB} 2$} & \multicolumn{2}{|c|}{ Error Modified 2-step } \\
\hline & $x_{1}(t ; \alpha)$ & $x_{2}(t ; \alpha)$ & $x_{1}(t ; \alpha)$ & $x_{2}(t ; \alpha)$ & $x_{1}(t ; \alpha)$ & $x_{2}(t ; \alpha)$ & $x_{1}(t ; \alpha)$ & $x_{2}(t ; \alpha)$ & $x_{1}(t ; \alpha)$ & $x_{2}(t ; \alpha)$ \\
\hline 0.0 & -0.9816 & 0.9816 & -0.9739 & 0.9739 & -0.9738 & 0.9738 & -0.0078 & 0.0078 & $-7.09 e-5$ & $7.09 \mathrm{e}-5$ \\
\hline 0.1 & -0.9312 & 0.9312 & -0.9239 & 0.9239 & -0.9238 & 0.9238 & -0.0074 & 0.0074 & $-6.72 e-5$ & $6.72 \mathrm{e}-5$ \\
\hline 0.2 & -0.8780 & 0.8780 & -0.8711 & 0.8711 & -0.8710 & 0.8710 & -0.0070 & 0.0070 & $-6.34 \mathrm{e}-5$ & $6.34 \mathrm{e}-5$ \\
\hline 0.3 & -0.8213 & 0.8213 & -0.8148 & 0.8148 & -0.8147 & 0.8147 & -0.0065 & 0.0065 & $-5.93 e-5$ & $5.93 e-5$ \\
\hline 0.4 & -0.7603 & 0.7603 & -0.7544 & 0.7544 & -0.7543 & 0.7543 & -0.0060 & 0.0060 & $-5.49 e-5$ & $5.49 \mathrm{e}-5$ \\
\hline 0.5 & -0.6941 & 0.6941 & -0.6886 & 0.6886 & -0.6886 & 0.6886 & -0.0055 & 0.0055 & $-5.01 e-5$ & $5.01 \mathrm{e}-5$ \\
\hline 0.6 & -0.6208 & 0.6208 & -0.6159 & 0.6159 & -0.6159 & 0.6159 & -0.0049 & 0.0049 & $-4.48 \mathrm{e}-5$ & $4.48 \mathrm{e}-5$ \\
\hline 0.7 & -0.5376 & 0.5376 & -0.5334 & 0.5334 & -0.5334 & 0.5334 & -0.0043 & 0.0043 & $-3.88 \mathrm{e}-5$ & $3.88 \mathrm{e}-5$ \\
\hline 0.8 & -0.4390 & 0.4390 & -0.4355 & 0.4355 & -0.4355 & 0.4355 & -0.0035 & 0.0035 & $-3.17 e-5$ & $3.17 \mathrm{e}-5$ \\
\hline 0.9 & -0.3104 & 0.3104 & -0.3079 & 0.3079 & -0.3079 & 0.3079 & -0.0025 & 0.0025 & $-2.24 \mathrm{e}-5$ & $2.24 \mathrm{e}-5$ \\
\hline 1.0 & 0.0000 & 0.0000 & 0.0000 & 0.0000 & 0.0000 & 0.0000 & 0.0000 & 0.0000 & 0.0000 & 0.0000 \\
\hline
\end{tabular}

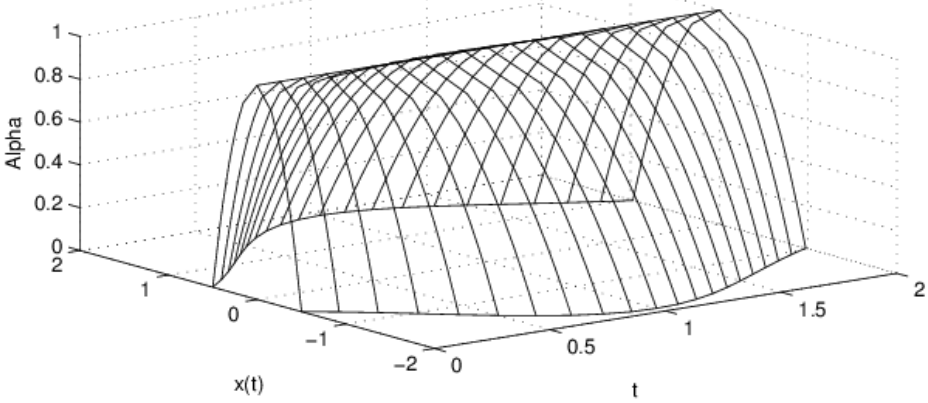

Fig 3 : The exact and approximation of fuzzy solution by modified two-step Simpson method $(\mathrm{h}=0.1)$
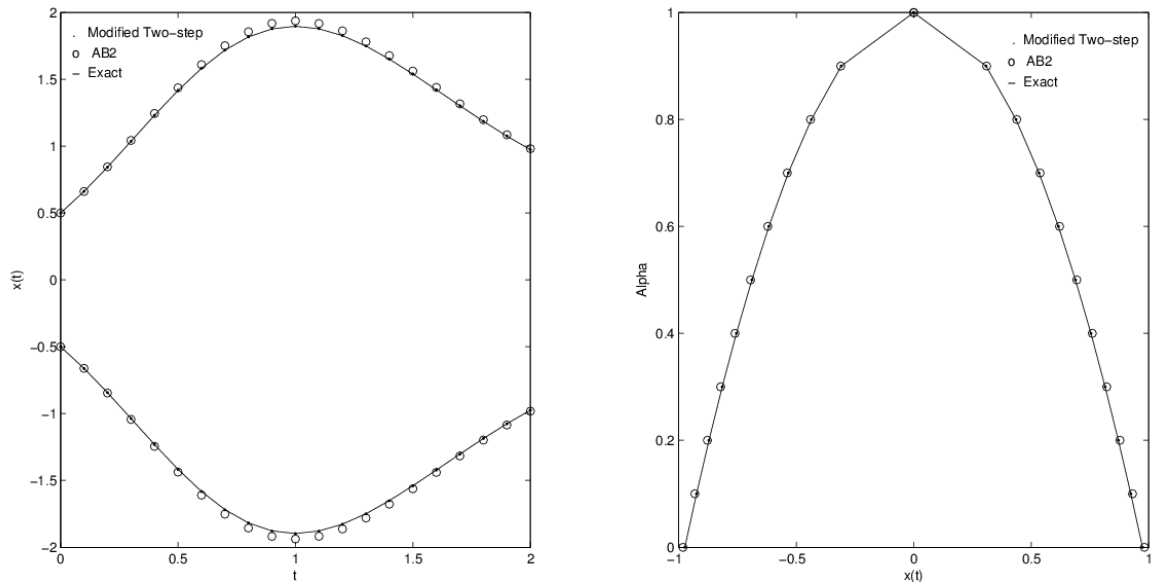

Fig 4 : Comparison between the exact, AB2, modified two-step Simpson method

The approximation fuzzy solutions by the proposed two-step method and Adams-Bashforth method of order two are given in Figure 3 and 4 respectively.

In this example, we compare the solution obtained by two-step method with the exact solution and the solution obtained by Adams-Bashforth method of order two. We have given the numerical values in Table 2 fixed value of $t_{20}=2$ and for different values of $\alpha$.

\section{Conclusion}

In this paper we used the modified two-step Simpson method for solving FIVP by taking into account the dependency problem in fuzzy computation. We compared the solutions obtained in two numerical examples. Two-step method provides as more accurate results than Adams-Bashforth method of order two. 


\section{Acknowledgements}

This work has been supported by “Tamilnadu State Council of Science and Technology”, Tamilnadu, India.

\section{References}

[1]. S. Abbasbandy, T. Allahviranloo, Numerical solutions of fuzzy differential equations by Taylor Method, Computational Methods in Applied Mathematics 2 (2002) 113-124.

[2]. S. Abbasbandy, T. Allahviranloo, Numerical solution of Fuzzy differential equation by Runge-Kutta method, Nonlinear Studies 11 (2004) $117-129$.

[3]. M. Ahamad, M. Hasen, A new approach to incorporate uncertainity into Euler method, Applied Mathematical Sciences 4(51) (2010) 2509-2520.

[4]. M. Ahamed, M. Hasan, A new fuzzy version of Euler's method for solving diffrential equations with fuzzy initial values, Sians Malaysiana 40 (2011) 651-657.

[5]. M. Ahmad, M. Hasan, Incorporating optimization technique into Zadeh's extension principle for computing non-monotone functions with fuzzy variable, Sains Malaysiana 40 (2011) 643-650

[6]. N. Z. Ahmad, H. K. Hasan, B. De Baets, A new method for computing continuous function with fuzzy variable, Journal of Applied Sciences 11(7) (2011) 1143-1149.

[7]. A. H. Alsonosi Omar, Y. Abu Hasan, Numerical solution of fuzzy differential equations and the dependency problem, Applied Mathematics and Computation 219 (2012) 1263-1272.

[8]. A. Bonarini, G. Bontempi, A Qualitative simulation approach for fuzzy dynamical models, ACM Trans. Model. Comput. Simulat. 4 (1994) 285-313.

[9]. R. Brent, Algorithms for Minimization without Derivatives, Dover Pubns 2002.

[10]. J. J. Buckley, T. Feuring, Fuzzy differential equations, Fuzzy Sets and Systems 110 (2000) 43-54.

[11]. D. Dubois, H. Prade, Towards fuzzy differential calculus part 3: differentiation, Fuzzy Sets and Systems 8 (1982) 225-233.

[12]. Kaleva Osmo, Fuzzy differential equations, Fuzzy Sets and Systems 24 (1987) 301-317.

[13]. O. Kaleva, Interpolation of fuzzy data, Fuzzy Sets and Systems 60 (1994) 63-70.

[14]. M. Ma, M. Friedman, A. Kandel, Numerical solutions of fuzzy differential equations, Fuzzy Sets and Systems 105 (1999) $133-138$

[15]. Mahmoud Mohseni Moghadam, Mohammad Shafie Dahaghin, Two-step methods for numerical solution of fuzzy differential equations.

[16]. R. E. Moore, Interval Analysis, Parentice-Hall, Englewood cliffs, N.J 1966.

[17]. S. Palligkinis, G. Papageorgiou, I. Famelis, Runge-Kutta methods for fuzzy differential equations, Applied Mathematics and Computation 209 (2009) 97-105.

[18]. M. Rostami, M. Kianpour, E. Bashardoust, A numerical algorithm for solving nonlinear fuzzy differential equations, The Journal of mathematics and Computer Sciences 2(4) (2011) 667-671

[19]. S. Seikkala, On the fuzzy initial value problem, Fuzzy Sets and Systems 24(3) (1987) 319-330

[20]. L. A. Zadeh, Fuzzy Sets, Information and Control 8 (1965) 338-353. 